\title{
Comment intégrer le développement durable dans un mégaprojet? Le cas de la Ville de Sept-Îles
}

\author{
Claude Villeneuve, Stéphanie Provost, David Tremblay et Hélène Côté ${ }^{1}$
}

\section{INTRODUCTION}

Sept-Îles longe ainsi une baie de $45 \mathrm{~km}^{2}$ dont l'entrée est protégée par un rempart naturel constitué des sept îles qui lui ont donné son nom. L'histoire de Sept-Îles est intimement liée à la richesse des ressources naturelles de son territoire. [...] Aujourd'hui, Sept-Îles est une ville moderne dotée de tous les équipements et services, ce qui en fait un centre urbain de première importance sur la Côte-Nord.

Située sur la Côte-Nord du Saint-Laurent, Sept-Îles compte environ 30000 habitants incluant la communauté innue. Stratégique pour son port en eau profonde où transitent des marchandises et matériaux nécessaires aux affaires de la grande industrie, elle est privilégiée par la beauté et la richesse de son territoire. Sept-Îles longe ainsi une baie de $45 \mathrm{~km}^{2}$ dont l'entrée est protégée par un rempart naturel constitué des sept îles qui lui ont donné son nom. L'histoire de Sept-Îles est intimement liée à la richesse des ressources naturelles de son territoire. D'abord lieu de rassemblement des Innus, ce milieu a tour à tour accueilli des trappeurs, des pêcheurs, puis des exploitants forestiers et miniers. Aujourd'hui, Sept-Îles est une ville moderne dotée de tous les équipements et services, ce qui en fait un centre urbain de première importance sur la Côte-Nord.

La ville fait actuellement l'objet d'un important potentiel de développement qui découle de la mise en œuvre de la volonté du Gouvernement du Québec d'exploiter les ressources naturelles situées au nord du $49^{\mathrm{e}}$ parallèle. Ces projets sont tous porteurs de développement économique, mais entraînent aussi des pressions accrues sur l'environnement et des impacts sur la communauté. La Ville souhaite dorénavant orienter son développement dans une perspective de développement durable surtout dans le contexte où la Loi du développement durable adoptée par l'Assem- blée nationale en 2006 doit à terme s'appliquer aux municipalités de toute la province.

Pour ce faire, consciente par son histoire des contraintes du développement de ce genre de projets et motivée par sa Corporation de protection de l'environnement de Sept-Îles (CPESI), la Ville a décidé de se doter d'un outil d'analyse de développement durable pour bonifier les projets et soutenir sa prise de décision dans le cadre de ses responsabilités et de sa planification du territoire. L'outil choisi est la grille d'analyse de développement durable (GADD) développé par la Chaire en éco-conseil de l'Université du Québec à Chicoutimi $^{2}$ (UQAC) et il a été expérimenté pour la première fois en 2012-2013 dans l'analyse du projet minier Arnaud en amont de sa présentation aux audiences du Bureau d'audiences publiques sur l'environnement du Québec (BAPE).

Créée en 1979, la Corporation de protection de l'environnement de Sept-îles (CPESI) est une corporation paramunicipale sans but lucratif. Elle vise à sensibiliser la population à l'environnement, au développement durable (DD) et à la protection des ressources. Concrètement, elle travaille à la création d'un " sentiment de compétence » chez la population par des activités ba-sées sur une approche qui favorise l'expérience sociale, la curiosité, l'interaction, l'innovation et l'apprentissage, et ce, en privilégiant des valeurs telles que l'intégrité, la responsabilité sociale, l'équité, l'éthique et le respect de l'environnement ${ }^{3}$.

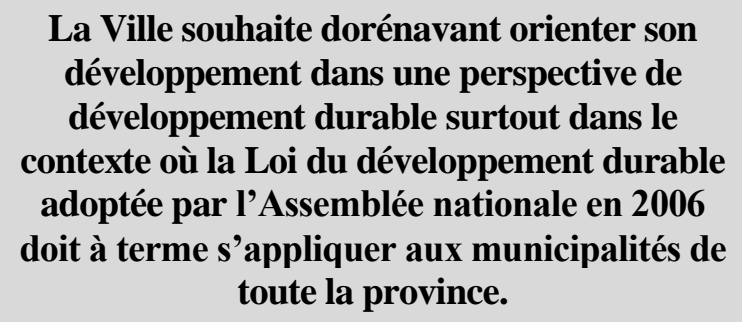

La Ville souhaite dorénavant orienter son développement dans une perspective de développement durable surtout dans le contexte où la Loi du développement durable adoptée par l'Assemblée nationale en 2006 doit à terme s'appliquer aux municipalités de toute la province. 


\section{MISE EN CONTEXTE}

\subsection{La grille d'analyse de développement durable (GADD)}

La GADD utilisée par la Chaire en éco-conseil de l'UQAC a été créée il y a plus de vingt ans pour répondre à des besoins d'analyse de projets et a évolué au cours de la dernière décennie pour devenir un outil plus large. Dans sa forme actuelle ${ }^{4}$, elle comporte 5 dimensions (éthique, environnementale, sociale, économique et gouvernance), 32 thèmes et 101 objectifs qui permettent à travers un mécanisme de pondération et d'évaluation d'identifier les forces et les faiblesses d'un projet, d'une politique d'un programme ou d'une stratégie, pour y prioriser des pistes de bonification. La pondération est une opération préalable à l'évaluation dans laquelle les analystes décident par consensus de l'importance relative d'un objectif donné dans le cadre du projet examiné. L'évaluation, pour sa part, constitue la note moyenne obtenue pour la performance des actions posées ou envisagées par le promoteur pour corriger ou atténuer les effets négatifs ou pour optimiser les retombées positives. Une performance faible dans un objectif prioritaire indique la nécessité d'améliorations urgentes. La GADD est un outil d'aide à la décision qui permet d'élargir les perspectives, d'encadrer le dialogue et de permettre de trouver des solutions appropriées au contexte local dans le respect de l'idéal du développement durable ${ }^{5}$. La grille d'analyse de développement durable a été utilisée dans de nombreux projets et contextes différents au Québec, au Canada aux États-Unis et dans plusieurs pays de la Francophonie. Elle a donné naissance à d'autres outils d'analyse, le plus récent étant la grille d'analyse de développement durable de la Francophonie 6 .

\subsection{Application de la grille au projet de Mine Arnaud}

Au Québec, les municipalités disposent de peu d'outils législatifs et surtout réglementaires pour se positionner sur les projets qui proposent de s'implanter sur leur territoire. Ces dernières doivent donc innover et adapter leurs stratégies et leurs actions. En 2012, la Ville de Sept-Îles a mandaté la CPESI pour mettre en œuvre une démarche exploratoire visant à doter la municipalité d'outils pour la guider dans l'encadrement de son développement dans une perspective de développement durable et dans le respect de la communauté. Le choix de la GADD par la CPESI a été motivé par le caractère collectif et inclusif de l'outil. En effet, cette grille d'analyse est construite de manière à répondre aux préoccupations des individus et collectivités, contrairement à la majorité qui sont destinées aux promoteurs de projets. Cela convenait aux dirigeants de la CPESI qui souhaitaient que les élus et l'administration municipale se dotent d'une compétence interne permettant de favoriser la prise de décision.

Cette nouvelle compétence s'est exercée pour la première fois sur le projet minier Arnaud ${ }^{7}$ La démarche visait à élargir la réflexion des acteurs du milieu, stimuler la discussion autour du projet en y intégrant des enjeux de développement durable, vérifier si les connaissances et l'analyse des besoins par le promoteur étaient suffisantes, vérifier quels éléments étaient sous-représentés ou manquants et procéder à une éducation des parties prenantes. Les enjeux de ce mégaprojet qui deviendrait à terme la plus grande mine à ciel ouvert en milieu périurbain au Canada affecteraient potentiellement la qualité de l'eau, de l'air et pourrait avoir des impacts sociaux encore hypothétiques.

\section{La grille d'analyse de développement durable a été utilisée dans de nombreux projets et contextes différents au Québec, au Canada aux États Unis et dans plusieurs pays de la Francophonie. Elle a donné naissance à d'autres outils d'analyse, le plus récent étant la grille d'analyse de développement durable de la Francophonie.}

Pour réaliser l'analyse, la CPESI a formé, avec la collaboration et le soutien de la Ville un comité d'analyse en développement durable (CADDVSI), incluant des intervenants provenant des secteurs social, économique, municipal et de la société civile. Ce comité autonome a pris connaissance de l'information disponible (étude d'impact et autres documents pertinents) avant de procéder à l'analyse. 
Compte tenu du biais environnemental qui résulte de la nature et de la mission de la CPESI et du caractère novateur de la démarche, la corporation a demandé l'encadrement et l'expertise de la Chaire en éco-conseil de l'UQAC. Le rôle des professionnels de la Chaire était d'accompagner le comité et la CPESI afin de s'assurer d'une utilisation rigoureuse de la grille lors de l'analyse du projet. Les représentants de la Chaire ne sont intervenus ni dans la pondération, ni dans l'évaluation, mais ont rédigé le rapport d'analyse de développement durable.

\section{ASPECTS THÉORIQUES}

Le développement durable est un paradigme qui fait référence à plusieurs dimensions dont les plus souvent citées sont l'environnement, l'économie et la dimension sociale, représentés sous forme de cercles à l'intersection desquels se situe la durabilité. Toutefois, en plus de 40 ans d'histoire, le concept s'est complexifié. Dans cet article, nous ferons appel à un modèle à cinq dimensions, ajoutant l'éthique et la gouvernance au modèle classique? .

Comme les autres industries extractives, le secteur minier est confronté à une multitude d'enjeux et de controverses remettant en cause sa durabilité dans ses dimensions économiques, sociales, environnementales et éthiques. La résolution des enjeux de gouvernance est, pour sa part, garante de la performance des entreprises dans chacune des quatre autres dimensions ${ }^{10}$. À l'heure actuelle, il est reconnu que la conciliation entre l'industrie minière et les exigences du développement durable représente un grand défi pour l'ensemble des parties prenantes. Une telle conciliation doit désormais se baser sur une approche englobante qui prend en compte l'ensemble des considérations de l'exploitation minière. Le débat sur cette problématique résulte d'abord de la nature non renouvelable des ressources minières, de leur répartition ponctuelle qui interpelle l'équité inter et intra générationnelle et des enjeux et impacts de leur exploitation qui touchent à la fois les humains (peuples autochtones, employés et leurs familles, femmes, etc.), les écosystèmes et les autres ressources naturelles (eau, air, sol, biodiversité).
Tout au long de l'exercice, le comité s'est efforcé d'effectuer une démarche transparente et ouverte dans laquelle les parties intéressées pouvaient intervenir sur une section consacrée au projet Mine Arnaud sur le portail Synapse ${ }^{8}$ géré par la Chaire. Toute documentation produite ou consultée par le comité ainsi que celle que des intervenants souhaitaient porter à son attention y étaient divulguées sans délai pour favoriser l'information et la participation du public.

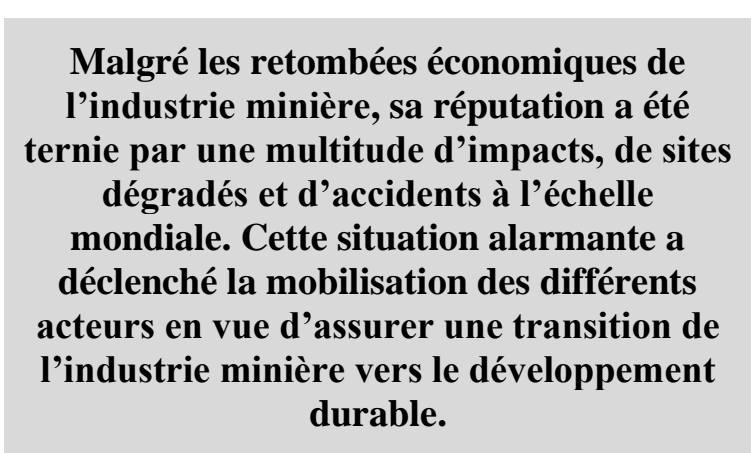

En effet, malgré les retombées économiques de l'industrie minière, sa réputation a été ternie par une multitude d'impacts, de sites dégradés et d'accidents à l'échelle mondiale. Cette situation alarmante a déclenché la mobilisation des différents acteurs en vue d'assurer une transition de l'industrie minière vers le développement durable. Cette mobilisation a profité de plusieurs conventions, pactes et protocoles internationaux ainsi que de l'émergence d'une multitude d'organismes de surveillance et de normalisation qui ont mis en place des lignes directrices, référentiels et codes de conduite pour les entreprises minières. Les revendications sociales prônées par la société civile, les syndicats et les ONG ont contribué également à un plaidoyer pour les droits des communautés touchées. Les médias ont eux aussi joué un grand rôle dans la divulgation des impacts et incidences de l'industrie minière ainsi que dans la sensibilisation des acteurs.

L'intérêt grandissant pour le développement durable trouve également écho, depuis près de 
deux décennies, dans les pratiques d'urbanisme et d'aménagement du territoire. La Loi québécoise sur le développement durable (LQDD), qui s'applique à l'appareil gouvernemental depuis son adoption en 2006, prévoit d'ailleurs à l'article 4 son éventuel élargissement aux administrations municipales pour qu'elles participent à l'effort de mise en œuvre du développement durable. Plusieurs villes québécoises intègrent partiellement ces nouvelles pratiques de développement durable dans leurs exercices de planification territoriale. Toutefois, à l'échéance de la stratégie québécoise de développement durable (2008-2013), force est de constater que peu de municipalités appliquent globalement les pratiques de l'urbanisme durable, à l'échelle de leur territoire. Les schémas d'aménagement et de développement durable et les plans d'urbanisme durable demeurent l'exception plutôt que la règle. Les pratiques urbanistiques qui ont, à long terme, des conséquences néfastes ${ }^{11}$ au niveau social, économique et environnemental sont encore légion ${ }^{12}$.

Dans ce contexte, l'établissement d'un mégaprojet minier dans les frontières de la Ville de Sept-Îles présente un intérêt certain pour explorer les conditions d'application du développement durable. L'utilisation de la GADD devait aussi pouvoir identifier des pistes de bonification qui pourraient rendre le projet plus compatible avec la volonté de développement durable manifestée par la Ville. Enfin, ce projet étant soumis à la procédure d'évaluation des impacts $\mathrm{du}$ Gouvernement du Québec et assujetti au BAPE, le résultat de l'analyse avec la GADD devait passer l'épreuve de l'examen par une autre équipe et une autre méthodologie, ce qui renforce l'intérêt académique de l'expérience.

\section{APPLICATION DE LA GRILLE DANS LE PROJET MINE ARNAUD}

Le tableau 1 donne une chronologie du projet minier Arnaud. On peut y constater que l'analyse a été produite entre le dépôt de l'étude d'impact et le mandat du BAPE, ce qui a permis de diffuser les résultats de l'analyse dans la communauté et de déposer le rapport d'analyse dans le processus d'examen de la commission.

\section{Tableau 1 - Chronologie du projet minier Arnaud}

\begin{tabular}{|l|l|}
\hline Dépôt de l'avis de projet par Mine Arnaud & 14 décembre 2010 \\
\hline Dépôt de l'étude d'impact par Mine Arnaud & 26 mars 2012 \\
\hline Lancement de la démarche d'analyse & 09 octobre 2012 \\
\hline Rencontres préparatoires (3) du CADDVSI & Du 15 octobre au 2 novembre 2012 \\
\hline Lecture de la documentation & Du 15 octobre au 4 décembre 2012 \\
\hline Portail public Mine Arnaud (synapse.ca) & Du 15 octobre 2012 au 31 mars 2013 \\
\hline Rencontre de pondération (CADDVSI) & 5 décembre 2012 \\
\hline Rencontre experts par la CADDVSI & 5 décembre 2012 \\
\hline Rencontre d'évaluation par le CADDVSI & $14-15$ janvier 2013 \\
\hline Rédaction du rapport d'analyse du CADDVSI & Du 20 janvier au 27 février 2013 \\
\hline Présentation du rapport & $18-19$ mars 2013 \\
\hline $\begin{array}{l}\text { Période d'information et de consultation } \\
\text { publique (BAPE) }\end{array}$ & Du 21 mai au 5 juillet 2013 \\
\hline Mandat d'audiences publiques (BAPE) & Du 26 aout au 20 décembre 2013 \\
\hline Dépôt public du rapport du BAPE & 19 février 2014 \\
\hline
\end{tabular}




\section{LES RÉSULTATS}

L'analyse de développement durable a été rendue publique en mars $2013^{13}$. L'évaluation globale du comité pour chacune des dimensions se situait dans la tranche supérieure de la catégorie «des projets qui répondent moyennement aux objectifs sans se démarquer des autres projets semblables, mais en y accordant tout de même une certaine attention ». Toutefois, plus de $50 \%$ des objectifs jugés indispensables ou nécessaires par le CADDVSI ont obtenu une évaluation moyenne ou faible. Treize pistes de bonification ont été identifiées comme prioritaires en raison de leur potentiel d'améliorer la performance du projet sur des enjeux ciblés et de leur effet potentiel sur plusieurs objectifs de la grille. Par exemple, des améliorations pour le partenariat avec les parties prenantes, le plan de fermeture de la mine, l'électrification des équipements ont été identifiées. Ces pistes correspondent souvent à des éléments soulevés par d'autres parties prenantes dans la période de préconsultation, mais ne représentent qu'une partie des pistes identifiées pour chacun des objectifs. Le rapport concluait que si elles étaient prises en considération par le promoteur, le projet serait fortement amélioré.

L'analyse de la revue de presse a aussi montré que l'ensemble des préoccupations soulevées par le public étaient couvertes par au moins l'un des 101 objectifs de la grille d'analyse. Le processus a donc évalué la globalité des enjeux identifiés. Vingt-sept grilles ont été complétées par des citoyens ou des groupes et portées à l'attention de la CPESI, cependant, aucune n'a été rendue publique à la demande des auteurs. Les pistes de bonification qui y ont été consignées ont cependant été recensées par les membres du CADDVSI et prises en considération dans l'analyse.

Une grille a été remplie par le promoteur et rendue publique. La figure 1 compare les deux évaluations. Parmi les actions identifiées par le promoteur, plusieurs correspondent à des pistes de bonification identifiées par le CADDVSI. Cette constatation permettait de conclure qu'un dialogue était toujours possible pour procéder à la bonification du projet dans une perspective de développement durable. Sans surprise, l'évaluation que le promoteur a faite de son propre projet est plus forte que celle du CADDVSI, mais il n'a pas été relevé d'incohérence entre les différences d'évaluation par dimension.

En plus de permettre de prendre des décisions éclairées basées sur un exercice logique s'inscrivant dans les principes de développement durable, le rapport d'analyse a pu alimenter la réflexion de la population en plus de service à mieux préparer la consultation publique du BAPE. En effet, outre la Ville de Sept-Îles, plusieurs organisations du milieu ont aussi utilisé ce rapport pour étayer leur mémoire déposé à la commission d'enquête : Cégep de Sept-Îles, Chambre de commerce de Sept-Îles, Développement économique Sept-Îles, etc.

Figure 1 - Comparaison des deux évaluations

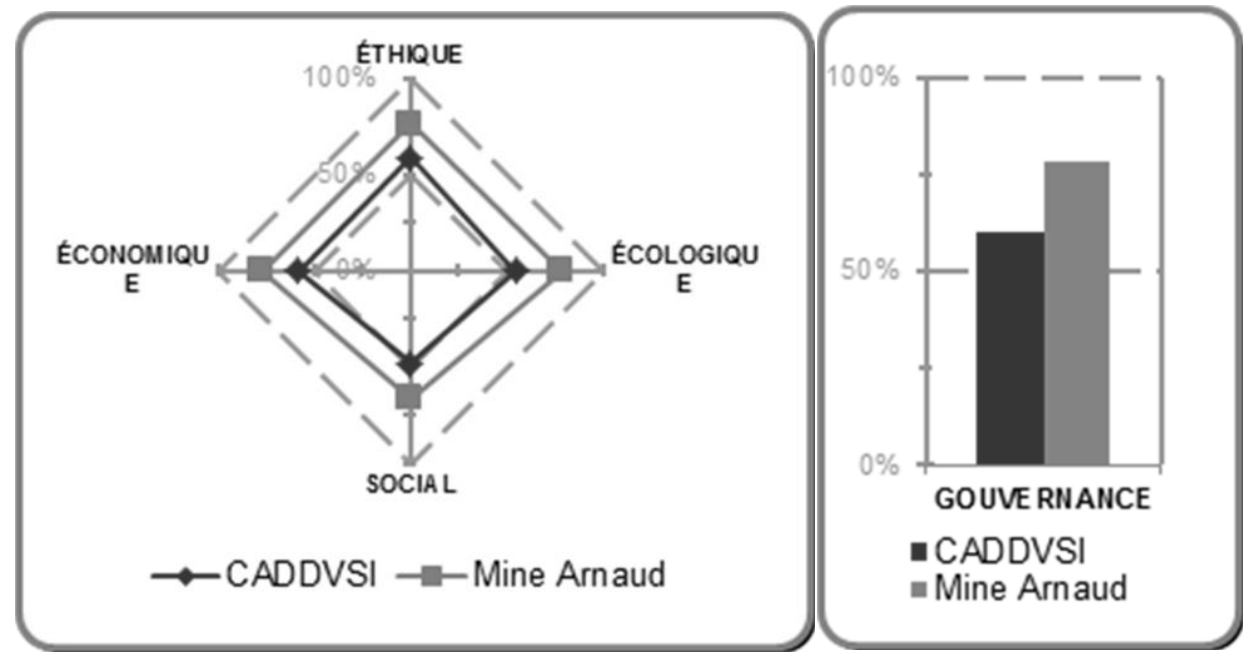


Le rapport a aussi été déposé en séance auprès de la commission d'enquête chargée de la consultation publique sur le projet Mine Arnaud par la CPESI. Pendant la première partie des audiences, la représentante de la CPESI a posé plusieurs questions en lien avec les pistes de bonification qui auraient pu être étudiées ou retenues par le promoteur pour améliorer la performance de son projet en termes de développement durable. Malheureusement, les réponses données (par exemple dans le document DA18) ${ }^{14}$ à la plupart de ces questions ont été jugées insatisfaisantes ou incomplètes par l'organisme. La CPESI a donc considéré que l'information apportée lors cette première partie n'a pas contribué à modifier de façon significative le portrait résultant de l'analyse de développement durable du CADDVSI. Tant le promoteur que les personnes ressources des différents ministères (MDDEFP, MRN, MSSS) n'ont pas été en mesure d'annoncer des engagements répondant à certaines propositions de bonification jugées indispensables pour une intégration durable dans la collectivité. Cette constatation a été reprise dans le mémoire de la CPESI.

Le rapport de la Commission du BAPE a été déposé au ministre du Développement durable,

\section{ANALYSE}

L'analyse de développement durable au projet minier Arnaud montre bien l'intérêt pour une Ville de se doter d'un tel outil. L'expérience a démontré qu'un comité de personnes issues du milieu pouvait maîtriser l'outil et faire valoir les résultats de l'analyse devant les autorités compétentes, facilitant ainsi l'aide à la décision.

L'analyse de développement durable au projet minier Arnaud montre bien l'intérêt pour une Ville de se doter d'un tel outil. L'expérience a démontré qu'un comité de personnes issues du milieu pouvait maîtriser l'outil et faire valoir les résultats de l'analyse devant les autorités compétentes, facilitant ainsi l'aide à la décision. Toutefois, certaines observations issues de l'expérience montrent que les effets positifs de la grille auraient pu être plus intéressants. En de l'Environnement, de la Faune et des Parcs du Québec le 20 décembre 2013. Il a été rendu public le 19 février 2014 et concluait: “ $A$ la lumière de ses travaux, la commission ne peut que constater que l'ensemble du dossier est incomplet et ne répond pas adéquatement aux enjeux relatifs à la contamination des eaux de surface et des eaux souterraines ainsi qu'aux risques de glissement de terrain et de tassement de sol. Des évaluations complémentaires sont donc requises puisqu'elles sont essentielles pour une juste appréciation des impacts. En conséquence, la commission d'enquête est d'avis que le projet de Mine Arnaud n'est pas acceptable dans sa forme actuelle" ${ }^{, 15}$.

Il est intéressant de noter que la conclusion du rapport de la commission relevait, pour justifier son jugement, des mêmes éléments qui avaient été retenus dans l'analyse de développement durable réalisée par le CADDVSI. Pour qu'il soit acceptable dans un contexte de développement durable, le projet d'une mine à ciel ouvert en périmètre urbain aurait dû obtenir des résultats bien supérieurs pour les 5 dimensions analysées.

effet, la polarisation des opinions était déjà bien campée lorsque la Ville a annoncé son intention de procéder à l'analyse. Cette polarisation a eu pour effet de rendre plus difficile la pleine participation du public. Maintenant que l'usage de l'outil est connu, l'analyse pourrait commencer dès l'annonce des projets, ce qui donnerait un cadre au questionnement et favoriserait un dialogue plus constructif entre les parties prenantes. En effet, la grille en permettant d'élargir les perspectives favorise la prise en compte de la complexité.

Une utilisation plus systématique de l'analyse de développement durable comme aide à la prise de décision au niveau municipal peut apporter des dividendes, non seulement pour l'analyse de mégaprojets, mais aussi pour des projets plus modestes, pour la planification du développement d'un quartier ou pour l'orientation de certaines politiques municipales sectorielles. Cependant, la 
grille telle qu'elle a été utilisée pour le projet minier Arnaud exige un certain investissement. Pour alléger le processus, les mêmes auteurs ont développé un outil simplifié « 32 questions pour le développement durable » qui pourrait donner lieu à une adaptation sectorielle pour rendre plus explicites les liens des stratégies, programmes et politiques municipaux avec les objectifs du développement durable.

\section{CONCLUSION}

Les mégaprojets comportent des enjeux particuliers qui sont en relation avec leurs impacts environnementaux, économiques et sociaux. Leur potentiel de susciter l'enthousiasme des développeurs économiques et les inquiétudes des citoyens est proportionnel à leur portée, surtout dans le domaine extractif. Pourtant, ces projets sont généralement justifiés par la satisfaction de besoins humains et la très grande majorité des promoteurs souhaitent en limiter les impacts négatifs sur le milieu d'accueil. En fournissant un cadre de réflexion plus large que les simples principes de la LDD et en permettant le dialogue sur la bonification des projets, l'utilisation d'un outil comme la GADD de la Chaire en éco-conseil peut contribuer à identifier à moindre frais un projet dont les paramètres présentent des lacunes en termes de développement durable. Il peut aussi servir à ouvrir un dialogue entre les parties prenantes pour l'amélioration du projet en lui permettant de mieux identifier et répondre à plus de besoins dans la communauté d'accueil. Eu égard aux efforts exigés des promoteurs pour présenter leurs projets, il apparaît que l'utilisation de tels outils en amont des projets représente une sécurité significativement plus intéressante de développement durable pour les communautés d'accueil.

\section{BIBLIOGRAPHIE ET NOTES}

${ }^{1}$ Claude Villeneuve (professeur), David Tremblay et Hélène Côté (professionnels de recherches) sont associés à la Chaire en éco-conseil de l'UQAC alors que Stéphanie Prévost travaille pour la Corporation de protection de l'environnement de Sept-Îles (CPESI).

${ }^{2}$ Villeneuve, C. et Riffon, O. (2011). Grille d'analyse de développement durable, Chaire en éco-conseil fichier Excel.
Dans une logique pluridisciplinaire favorisée par la pensée complexe, l'utilisation de la grille d'analyse a été profitable pour la CPESI à plusieurs niveaux. Il a été salutaire d'identifier les enjeux prioritaires en lien avec le projet de Mine Arnaud puisqu'on devra créer des carrefours de discussion, entre autres entre l'écologie et l'économie, l'ingénierie et la gouvernance.

${ }^{3}$ Voir le lien suivant : http://ville.sept-iles.qc.ca/ environnement.

${ }^{4}$ Voir Villeneuve C. et Riffon O. (2011). Grille d'analyse de développement durable, Chaire en écoconseil fichier Excel.

${ }^{5}$ Villeneuve, C., O. Riffon, J.R. Wells et V. Grégoire (2009) «Une grille d'analyse pour le développement durable », Encyclopédie du développement durable, \# 85, Éditions des Récollets.

${ }^{6}$ IFDD, (2014). Grille d'analyse de développement durable de la Francophonie, fichier Excel.

${ }^{7}$ Voir le site suivant: www.minearnaud.com.

${ }^{8}$ Voir le site suivant : http://synapse.uqac.ca.

${ }^{9}$ Voir Villeneuve, C. (1998). Qui a peur de l'an 2000? Guide d'éducation relative à l'environnement pour le développement durable, UNESCO et Éditions Multimonde.

${ }^{10}$ Voir Bensalah, N., Ferrand, D. et Villeneuve, C. (2014). «Les mines et le développement durable : controverses et défis », CIM Journal, (à paraître).

${ }^{11}$ Pensons seulement à l'étalement urbain, à la déstructuration des territoires agricoles, à la fragmentation des usages ou à la trop grande place accordée à l'automobile.

${ }^{12}$ Voir Riffon, O. et Villeneuve, C. (2013). «Trois défis pour un urbanisme durable au Québec », Urbanité, numéro automne 2013, p. 50-54 et Simard, M. (2013). «L'urbanisme durable au Québec : Plaidoyer en faveur d'un cadre législatif incitatif », Organisations et territoires, vol. 22, $\mathrm{n}^{\circ} .3, \mathrm{p} .61-69$.

${ }^{13}$ Voir Tremblay, D., Bensalah, N., Cote, H. et Villeneuve, C. (2013): Rapport de la démarche d'analyse de développement durable du projet minier Arnaud selon la grille d'analyse de la Chaire en écoconseil. Rapport présenté à la Corporation de protection de l'environnement de Sept-îles (CPESI).

${ }^{14}$ Voir le site http://www.bape.gouv.qc.ca/sections/ mandats/mine_apatite_sept-iles/documents/liste_docDA-DB-DC.htm\#DA.

${ }^{15}$ Voir BAPE (2014). Projet d'ouverture et d'exploitation d'une mine d'apatite à Sept-Îles, rapport d'enquête et d'audiences publiques \# 301, 215 pages. 


\section{FORMULAIRE D'ABONNEMENT}

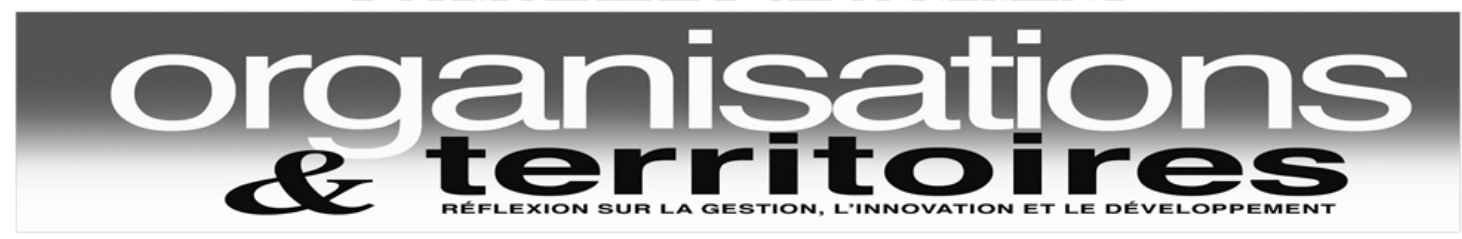

Je désire m'abonner à la revue Organisations et territoires et je joins un chèque au montant de :

Nouvel abonné $(\sqrt{ }): \square \quad$ Renouvellement $(\sqrt{ }): \square \quad$ Date :

Abonnement institutionnel (6 numéros) 103,48 \$ (Toutes taxes incluses)

Abonnement individuel (6 numéros) 66,69\$ (Toutes taxes incluses)

Abonnement étudiant (6 numéros) 48,29 \$ (Tx inc.) (Envoyer photocopie carte étud.)

Abonnement gouvernemental (6 numéros) $\quad 90,00 \$ \quad$ (Fournir certificat d'exemption des taxes)

Abonnement hors Canada (6 numéros) $145,00 \$ \quad(110 \$+35 \$$ frais de poste) (sans taxes)

Tous les abonnements sont d'une durée de deux ans

- J'aimerais que mon abonnement débute avec le DERNIER numéro paru, soit :

Volume $\mathrm{N}^{\circ}$

- J'aimerais que mon abonnement débute avec le PROCHAIN numéro à paraitre, soit :

Volume $\mathrm{N}^{\circ}$

Faites-nous parvenir vos coordonnées :

Nom :

Organisation :

Adresse :

Téléphone :

Télécopieur :

Courriel :

Adresse d'expédition: $\quad$ Revue Organisations et territoires

Université du Québec à Chicoutimi

555, boulevard de 1'Université, bureau H6-1430

Chicoutimi (Québec) CANADA G7H 2B1

Téléphone : (418) 545-5011, poste 4530 Télécopieur : (418) 693-9072

Courriel : revueot@uqac.ca Site Web : www.uqac.ca/revueot 